\title{
Detection and discrimination of influenza B Victoria lineage deletion variant viruses by real-time RT-PCR
}

Bo Shu ${ }^{1}$, Marie K Kirby ${ }^{1,2}$, Christine Warnes ${ }^{1}$, Wendy M Sessions ${ }^{1}$, William G Davis ${ }^{1,2}$, Ji Liu ${ }^{1,3}$, Malania M Wilson ${ }^{1}$, Stephen Lindstrom ${ }^{4}$, David E Wentworth ${ }^{1}$, John R Barnes ${ }^{1}$

1. Virology, Surveillance and Diagnostic Branch, Influenza Division, Centers for Disease Control and Prevention, Atlanta, United States

2. Battelle Memorial Institute, Atlanta, United States

3. Chickasaw Nation Industries, Inc., Atlanta, United States

4. Respiratory Virus Branch, Division of Viral Diseases, Centers for Disease Control and Prevention, Atlanta, United States

Correspondence: John R Barnes (fzq9@cdc.gov)

Shu Bo, Kirby Marie K, Warnes Christine, Sessions Wendy M, Davis William G, Liu Ji , Wilson Malania M, Lindstrom Stephen, Wentworth David E, Barnes John R. Detection and discrimination of influenza B Victoria lineage deletion variant viruses by real-time RT-PCR. Euro Surveill. 2020;25(41):pii=1900652. https://doi. org/10.2807/1560-7917.ES.2020.25.41.1900652

Article submitted on 24 Oct 2019 / accepted on 15 Apr 2020 / published on 15 Oct 2020

Background: During the 2016/17 influenza season, influenza B/VIC lineage variant viruses emerged with two $\left(\mathrm{K}_{162} \mathrm{~N}_{163}\right)$ or three $\left(\mathrm{K}_{162} \mathrm{~N}_{163} \mathrm{D}_{164}\right)$ amino acid (aa) deletions in the haemagglutinin (HA) protein. There are currently five antigenically distinct $\mathrm{HA}$ proteins expressed by co-circulating influenza $B$ viruses: $B /$ YAM, B/VIC V1A (no deletion), B/VIC V1A-2DEL (2 aa deletion) and two antigenically distinguishable groups

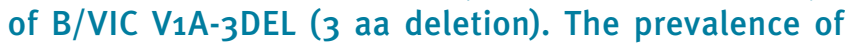
these viruses differs across geographical regions, making it critical to have a sensitive, rapid diagnostic assay that detects and distinguishes these influenza B variant viruses during surveillance. Aim: Our objective was to develop a real-time RT-PCR (rRT-PCR) assay for detection and discrimination of influenza $B$ / VIC lineage variant viruses. Methods: We designed a diagnostic assay with one pair of conserved primers and three probes specific to each genetic group. We used propagated influenza B/VIC variant viruses and clinical specimens to assess assay performance. Results: This rRT-PCR assay detects and distinguishes

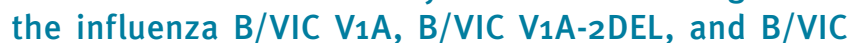
V1A-3DEL variant viruses, with no cross-reactivity. This assay can be run as a multiplex reaction, allowing for increased testing efficiency and reduced cost. Conclusion: Coupling this assay with the Centers for Disease Control and Prevention's Human Influenza Virus Real-Time RT-PCR Diagnostic Panel Influenza B Lineage Genotyping Kit results in rapid detection and characterisation of circulating influenza $B$ viruses. Detailed surveillance information on these distinct influenza B variant viruses will provide insight into their prevalence and geographical distribution and could aid in vaccine recommendations.

\section{Introduction}

Influenza B viruses co-circulate with influenza A strains during the annual influenza season, contributing to overall mortality and morbidity of influenza epidemics. In the 1980 s, influenza B viruses evolved into two genetically and antigenically distinct lineages represented by $\mathrm{B} /$ Victoria/2/1987 (VIC) and B/ Yamagata/16/1988 (YAM) that co-circulate in human hosts during influenza seasons worldwide [1-5]. During the 2016/17 influenza season, the United States (US) Centers for Disease Control and Prevention (CDC) detected influenza B/VIC viruses in the US that were antigenically distinct from the World Health Organization (WHO)-recommended vaccine virus, B/ Brisbane/6o/2008 (VIC) [6]. Sequencing analysis confirmed that these viruses have a deletion of 6 nucleotides ( $\mathrm{nt}$ ) in the haemagglutinin ( $\mathrm{HA})$ gene resulting in a 2 amino acid (aa) deletion at positions 162 and 163 (corresponding to nt positions 529-534) [6]. This B Victoria lineage 2 aa deletion virus ( $\mathrm{V} 1 \mathrm{~A}-2 \mathrm{DEL} ; \mathrm{K}_{162} \mathrm{~N}_{163}$ deletion) has since spread and been detected worldwide. Two $B /$ VIC lineage variants with a deletion of 3 aa $\left(V_{1} A-3 D E L\right)$ at aa positions $162-164\left(\mathrm{~K}_{162} \mathrm{~N}_{163} \mathrm{D}_{164}\right.$, i.e. nt positions 529-537) emerged shortly after via parallel evolution. The $V_{1} A-3 D E L$ viruses have since been detected in Asia, Africa, Europe and America [7]. There are currently five genetically distinct $\mathrm{HA}$ genes that yield five antigenically distinct influenza $B$ viruses that are co-

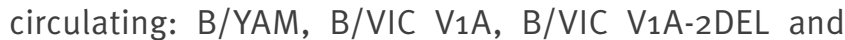
two groups of $B /$ VIC V1A-3DEL (Figure) [8].

The most effective method for prevention and control of influenza infection is vaccination $[3,4,9]$. Licensed seasonal vaccines are updated annually and the WHO makes recommendations on the composition of influenza virus vaccines on the basis of surveillance, laboratory and clinical observations [3]. This process 


\section{FIGURE}

Evolutionary relationships among influenza B Victoria and Yamagata haemagglutinin genes

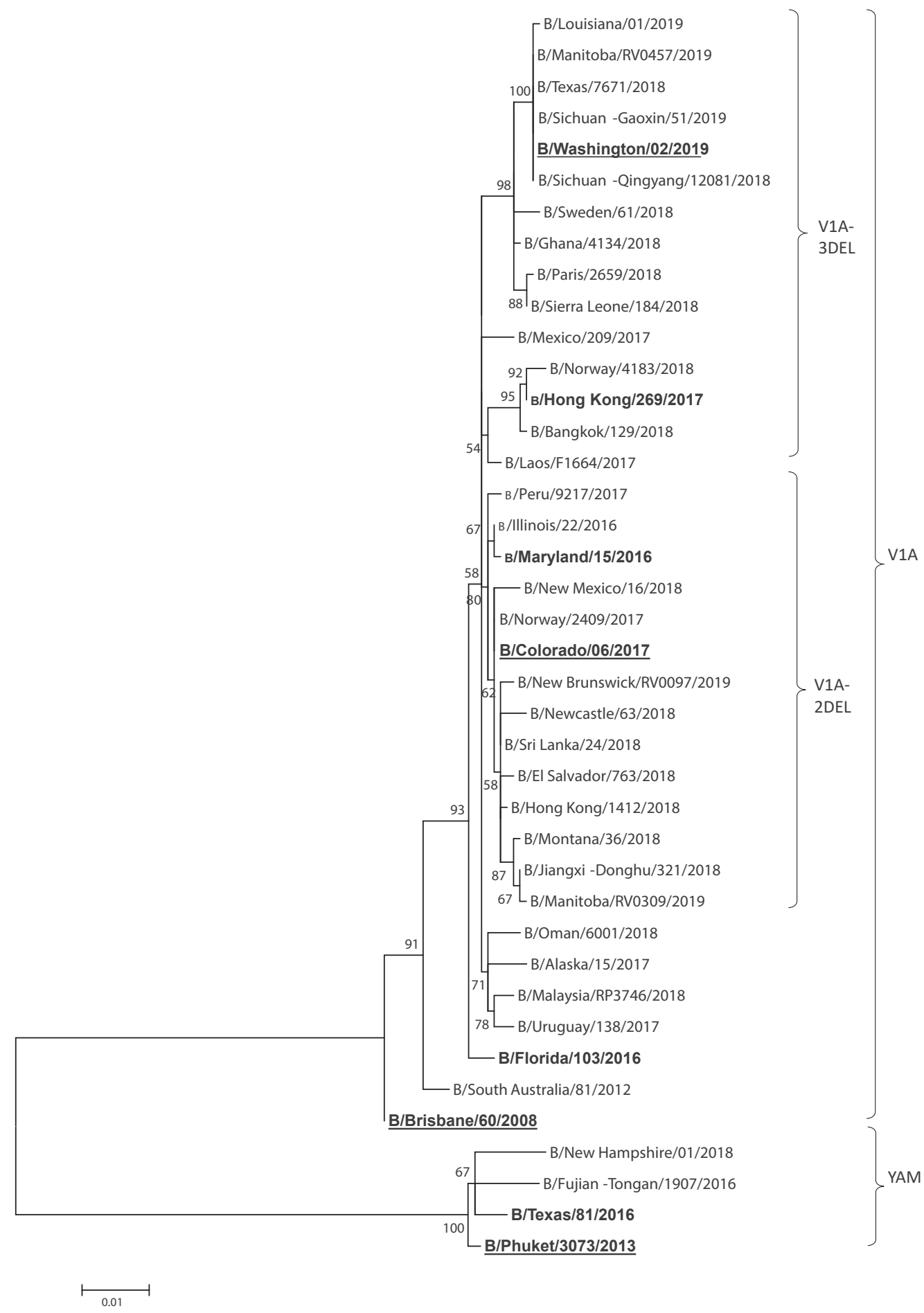

HA: haemagglutinin.

HA (coding sequence) phylogenetic tree with representative viruses of the genetic groups of B/Victoria and B/Yamagata lineages of related viruses; V1A, V1A-2DEL, V1A-3DEL and YAM are indicated by the bars on the right. The viruses evaluated in this study are shown in bold and influenza B vaccine viruses are underlined. HA sequences used in this analysis are detailed in Supplementary Table $\mathrm{S} 1$. Phylogenetic analysis was performed using Molecular Evolutionary Genetics Analysis software (MEGA, version 7.0). The evolutionary history was inferred using the maximum likelihood method. The evolutionary distances were computed using the Tamura-Nei method. The bootstrap value (1,000 replicates) of the HA gene is shown next to the branches. 
TABLE 1

Primer and probe sequences for the Centers for Disease Control and Prevention influenza B/Victoria lineage deletion detection assay

\begin{tabular}{|l|c|c|}
\hline Primer/probe & Sequence 5'-3' & Nucleotide position $^{\mathrm{a}}$ \\
\hline Forward primer & GAT TYT TYG CAA TGG CTT G & $497-518$ \\
\hline Vic 2_Del probe & CGT CCC AGA CAA AAA & $522-542$ \\
\hline Vic 3_Del probe $^{\mathrm{c}}$ & TCC CAA AAA AC AAAdACA & $524-544$ \\
\hline Vic No_Del probe $^{\mathrm{b}}$ & TTG TCG TTT GGG AC & $539-523$ \\
\hline Reverse primer $^{\mathrm{d}}$ & CCT TCT GTA CAA AYG TAT GGT ACT TC & $596-571$ \\
\hline
\end{tabular}

HA: haemagglutinin; Vic: Victoria.

a Nucleotide positions are according to HA gene of the B/Brisbane/60/2008(CY073893) strain.

b The BHQPlus probe has FAM at the 5 ' end, BHQ1 at the 3 ' end and employs C-5 propynyl-dC (pdC) for dC and C-5 propynyl-dU (pdU) for dT substitutions (Glen Research Corporation, Virginia, US).

' The BHQ and LNA probes have FAM at the 5'end, BHQ1 at the 3 send.

${ }^{\mathrm{d}}$ A triplet of locked nucleic acid residues.

\section{TABLE 2}

Haemagglutination inhibition reactions of influenza B viruses

\begin{tabular}{|c|c|c|c|c|c|}
\hline \multirow{3}{*}{ Influenza B virus strain } & \multicolumn{5}{|c|}{ Ferret antiserum } \\
\hline & \multirow{2}{*}{$\frac{\mathrm{V}_{1} \mathrm{~A}}{\mathrm{~B} / \text { Brisbane } / 60 / 200}$} & \multirow{2}{*}{$\frac{\text { V1A-2DEL }}{\text { B/Maryland/15/2016 }}$} & \multicolumn{2}{|c|}{ V1A-3DEL } & \multirow{2}{*}{$\begin{array}{c}\text { YAM } \\
\text { B/Phuket/3073/2013 }\end{array}$} \\
\hline & & & $\begin{array}{c}\text { B/Hong } \\
\text { Kong/286/2017 }\end{array}$ & $\mathrm{B} /$ Washington/02/2019 & \\
\hline \multicolumn{6}{|l|}{$V_{1} A$} \\
\hline B/Brisbane/60/2008 & 2,560 & 10 & 40 & 40 & $<10$ \\
\hline \multicolumn{6}{|l|}{ V1A-2DEL } \\
\hline $\mathrm{B} /$ Maryland/15/2016 & 80 & 160 & 10 & 20 & $<10$ \\
\hline B/Colorado/06/2017 & 80 & 160 & 10 & 10 & $<10$ \\
\hline \multicolumn{6}{|l|}{ V1A-3DEL } \\
\hline B/Hong Kong/286/2017 & 320 & 40 & 320 & 80 & $<10$ \\
\hline B/Hong Kong/269/2017 & 80 & 20 & 160 & 40 & $<10$ \\
\hline $\mathrm{B} /$ Washington/02/2019 & 40 & 40 & 40 & 320 & $<10$ \\
\hline \multicolumn{6}{|l|}{ Yamagata } \\
\hline B/Phuket/3073/2013 & $<10$ & $<10$ & $<10$ & $<10$ & 640 \\
\hline
\end{tabular}

Viruses used in this study are shown in bold and numbers in bold indicate homologous haemagglutination inhibition titres.

occurs twice a year, in February for the northern hemisphere and in September for the southern hemisphere. It is important to identify the most prevalent influenza A subtype and $B$ lineage viruses through influenza surveillance for influenza vaccine selection, especially for people who may not have been exposed to a subtype/lineage of influenza virus [10]. Identifying the optimal viruses to include in the next season's influenza vaccines is a significant challenge for the influenza surveillance network known as the Global Influenza Surveillance and Response System (GISRS). A diagnostic assay that allows for rapid identification of these genetically and antigenically distinct influenza $B$ viruses is beneficial to understanding how the prevalence of these viruses varies in different geographic regions of the world and will provide data that could guide relevant vaccine strain selection.

Here we describe a real-time RT-PCR (rRT-PCR)-based assay for detection and discrimination of influenza $B /$ VIC lineage deletion variants (Vic deletion assay). This assay is currently the only available method, to our knowledge, that can distinguish these B/VIC genetic groups, outside of a pyrosequencing technique and a conventional RT-PCR method, both recently published $[11,12]$.

\section{Methods}

\section{Influenza viruses, antigenic analysis and RNA extraction}

Influenza viruses tested in this study were grown to high titre in either Madin-Darby canine kidney (MDCK) cells or embryonated chicken eggs (ECE) [13]. Infectious virus in culture supernatants or allantoic fluids was measured by $50 \%$ tissue culture infectious dose $\left(\mathrm{TCID}_{50} / \mathrm{mL}\right)$ or $50 \%$ egg-infectious dose $\left(E_{50} / \mathrm{mL}\right)$, respectively [14]. Influenza $B$ virus isolates used to evaluate analytical performance included $B /$ Maryland/15/2016 and B/Colorado/6/2017 (V1A-2DEL), $\mathrm{B} /$ Hong Kong/269/2017 and B/Washington/02/2019 (V1A-3DEL) as well as no-deletion viruses $B /$ 
Assay limit of detection determined using quantified influenza B/Victoria lineage viruses in comparison with the quantified synthetic RNA (tested in triplicate)

\begin{tabular}{|c|c|c|c|c|c|c|c|c|c|c|}
\hline \multirow[b]{2}{*}{$\begin{array}{l}\text { RNA } \\
\text { copies }^{a}\end{array}$} & \multirow{2}{*}{$\begin{array}{c}\text { Ct Value } \\
\text { InfB }\end{array}$} & \multirow{2}{*}{$\begin{array}{c}\text { Virus titre } \\
\text { (TCID50/ } \\
\text { mL) }\end{array}$} & \multicolumn{2}{|c|}{ Ct Value $^{b}$} & \multirow{2}{*}{$\begin{array}{l}\text { Virus } \\
\text { titre } \\
\text { (EID50/ } \\
\mathrm{mL} \text { ) }\end{array}$} & \multicolumn{2}{|c|}{ Ct Value $^{b}$} & \multirow{2}{*}{$\begin{array}{c}\text { Virus titre } \\
\text { (TCID50/ } \\
\text { mL) }\end{array}$} & \multicolumn{2}{|c|}{ Ct value $^{b}$} \\
\hline & & & $\operatorname{lnfB}$ & VIC No_Del & & $\operatorname{lnfB}$ & VIC 2_Del & & InfB & VIC 3_Del \\
\hline \multicolumn{2}{|c|}{ Armored RNA } & \multicolumn{3}{|c|}{ B/Florida/103/2016 } & \multicolumn{3}{|c|}{ B/Maryland/15/2016 } & \multicolumn{3}{|c|}{ B/Washington/2/2019 } \\
\hline 5,000 & $26.38 \pm 0.17$ & $10^{3.3}$ & $26.23 \pm 0.59$ & $24.06 \pm 0.09$ & $10^{4.5}$ & $26.17 \pm 0.32$ & $24.53 \pm 0.36$ & $10^{1.2}$ & $27.64 \pm 0.79$ & $28.85 \pm 1.65$ \\
\hline 500 & $29.90 \pm 0.27$ & $10^{2.3}$ & $30.53 \pm 0.56$ & $28.38 \pm 0.09$ & $10^{3.5}$ & $29.60 \pm 0.91$ & $28.70 \pm 0.14$ & $10^{0.2}$ & $31.70 \pm 0.77$ & $34.41 \pm 1.75$ \\
\hline 50 & $33.14 \pm 0.22$ & $10^{1.3}$ & $34.77 \pm 0.98$ & $31.80 \pm 0.45$ & $10^{2.5}$ & $35.19 \pm 1.06$ & $32.60 \pm 0.26$ & $10^{-0.2}$ & $33.45 \pm 0.38$ & $37 \cdot 31 \pm 0.15$ \\
\hline 5 & $35.12 \pm 0.92$ & $10^{0.3}$ & $35.60 \pm 1.16$ & $35.35 \pm 0.17$ & $10^{1.5}$ & $36.14 \pm 0.32$ & $37.22 \pm 0.89$ & & & \\
\hline
\end{tabular}

Ct: cycle threshold; EID: egg-infectious dose; InfB: universal influenza B assay; TCID: tissue culture-infectious dose; VIC: Victoria.

a The RNA material (Armored RNA Quant CDC-9, received from AsuraGen,Inc.) included the highly conserved InfB primer/probe sequence of the NS gene region, derived from the influenza B/Victoria lineage virus B/Colorado/06/2017(EPI 969380). The reference virus sequences in this region were $100 \%$ identical to Armored RNA Quant CDC-9.

${ }^{b}$ Mean \pm standard deviation.

The virus titres and RNA copies at the level of the assay's limit of detection are highlighted in bold.

\section{TABLE $4^{*}$}

Analytical specificity (exclusivity) testing with influenza B Victoria and Yamagata lineage viruses

\begin{tabular}{|c|c|c|c|c|c|c|c|}
\hline \multirow{2}{*}{ Influenza B virus } & \multirow{2}{*}{ Lineage } & \multirow{2}{*}{ Genetic group } & \multirow{2}{*}{$\begin{array}{l}\text { Infectious titre } \\
\text { (ID } 50 / \mathrm{mL})\end{array}$} & \multicolumn{4}{|c|}{ Ct value } \\
\hline & & & & $\operatorname{lnfB}$ & VIC 2_Del & Vic 3_Del & VIC No_Del \\
\hline $\mathrm{B} /$ Maryland/15/2016 & VIC & $\mathrm{V}_{1} \mathrm{~A} 2$-DEL & $10^{8.5}$ a & 9.21 & 9.52 & Negative & Negative \\
\hline $\mathrm{B} /$ Colorado/6/2017 & VIC & V1A 2-DEL & $10^{9.4 \mathrm{a}}$ & 10.14 & 10.18 & Negative & Negative \\
\hline B/Hong Kong/269/2017 & VIC & V1A 3-DEL & $10^{4.2 \mathrm{~b}}$ & 14.92 & Negative & 16.01 & Negative \\
\hline $\mathrm{B} /$ Washington/02/2019 & VIC & $\mathrm{V}_{1} \mathrm{~A}_{3}$-DEL & $10^{8.2 \mathrm{a}}$ & 15.61 & Negative & 17.29 & Negative \\
\hline B/Florida/103/2016 & VIC & $\mathrm{V}_{1} \mathrm{~A}$ & $10^{6.3 \mathrm{~b}}$ & 10.96 & Negative & Negative & 11.80 \\
\hline $\mathrm{B} /$ Brisbane/60/2008 & VIC & $\mathrm{V}_{1} \mathrm{~A}$ & $10^{7.9} \mathrm{a}$ & 15.67 & Negative & Negative & 16.27 \\
\hline B/Phuket/3073/2013 & YAM & Not applicable & $10^{7.9} \mathrm{a}$ & 17.54 & Negative & Negative & Negative \\
\hline $\mathrm{B} /$ Texas/81/2016 & YAM & Not applicable & $10^{8.3 \mathrm{a}}$ & 13.40 & Negative & Negative & Negative \\
\hline
\end{tabular}

Ct: cycle threshold; EID: egg-infectious dose; InfB: universal influenza B assay; TCID: tissue culture-infectious dose; VIC: Victoria; YAM: Yamagata.

a Data represent EID50/mL.

${ }^{b}$ Data represent $\mathrm{TCID}_{50} / \mathrm{mL}$.

Florida/103/2016 and B/Brisbane/60/2008 (V1A). We also included YAM lineage viruses represented by $B /$ Phuket/3073/2013 and B/Texas/81/2016.

Influenza B virus lineages were confirmed using antigenic characterisation by $\mathrm{HI}$ assay on MDCK-propagated virus isolates and genetic sequence analysis. The antigenic characteristics of virus isolates were determined by $\mathrm{HI}$ tests using post-infection ferret sera raised against representative human influenza $B$ viruses. The $\mathrm{HI}$ test was performed as described previously [15].

Viral RNA was extracted from $100 \mu \mathrm{L}$ of supernatant or allantoic fluid and eluted into $100 \mu \mathrm{L}$ of RNA elution buffer using a MagNA Pure Compact RNA isolation kit on a MagNA Pure Compact instrument (Roche Applied Science) [16].

Primers and probes for the B/Victoria lineage deletion detection assay

Oligonucleotide primers and probes for the Vic deletion assay were designed based on available nucleotide sequence data from the GenBank database of the National Center for Biotechnology Information (NCBI) and the Global Initiative on Sharing All Influenza Data (GISAID).

The Vic deletion assay includes a single set of conserved amplification primers and three deletion-specific dual-labelled hydrolysis probes, including VIC 2_Del, Vic 3_Del and Vic No_Del probes. The three probes, targeted to the deletion region of the $\mathrm{HA}$ gene of influenza $B$ viruses, were designed to specifically detect and differentiate $V_{1} A-2 D E L, V_{1} A-3 D E L$ and the $V_{1} A$ genetic group (no-deletion) viruses (B/ VIC No_Del), respectively (Table 1). The Vic 2_Del and Vic No_Del probes were designed using Blackhole Quencher(BHQ)plus dual-labelled hydrolysis probes (BHQplus) that were labelled at the 5'-end with the reporter molecule 6-carboxyfluorescein (FAM) or Hex and CAL Fluor Red 610 and with $\mathrm{BHQ}_{1}$ (FAM or Hex) or BHQ2 (CAL Fluor Red 610) at the 3'-end. The Vic 3_Del probe was labelled at the 5'-end with the reporter molecule 6-carboxyfluorescein (FAM) and BHQ1 at the 


\section{TABLE 5}

Clinical performance of the influenza $\mathrm{B} /$ Victoria lineage deletion detection assay: detection of $\mathrm{B} /$ Victoria lineage specimens compared with sequencing results

\begin{tabular}{|l|c|c|c|}
\hline \multirow{2}{*}{ B/VIC deletion assay positive } & \multicolumn{4}{c|}{ Sequencing results } \\
\cline { 2 - 4 } & V1A & V1A-2DEL & V1A-3DEL \\
\hline Vic No_Del $(n=27$ positive samples) & 27 & 0 & 0 \\
\hline Vic 2_Del $(n=30$ positive samples $)$ & 0 & 30 & 0 \\
\hline Vic 3_Del $(n=20$ positive samples $)$ & 0 & 0 & 20 \\
\hline
\end{tabular}

Vic: Victoria.

${ }^{\text {a }}$ Genetic groups were determined by genetic analysis.

3'-end, and included a triplet of locked nucleic acids (LNA) $[17,18]$ that centred on the mismatch bases from $V_{1} A-2 D E L$ and $V_{1} A$ viruses.

We further evaluated primer and probe sequence specificity by sequence analysis of 51,759 gene segments available in the NCBI or GISAID databases. Primers were designed to have annealing temperatures of $\mathrm{ca}$ $60^{\circ} \mathrm{C}$ and probes were designed to have annealing temperatures of $\mathrm{ca} 68^{\circ} \mathrm{C}$ using PrimerExpress 3.0 software (Applied Biosystems, Foster City, US). Primers were synthesised by the Biotechnology Core Facility at the $C D C$.

\section{rRT-PCR reaction conditions}

Reaction conditions for rRT-PCR were based upon the US Food and Drug Administration (FDA)-approved CDC Human Influenza Virus Real-Time RT-PCR Diagnostic Panel (Flu rRT-PCR Dx Panel) $[16,19,20]$. PCR reaction parameters of the Vic deletion assay were optimised using Invitrogen SuperScript III Platinum One-Step quantitative RT-PCR (qRT-PCR) (Single-plex) Kits (catalog no. 11732088, Life Technologies, Grand Island, US) and TaqPath qPCR Multiplex Master Mix (Triplex) kits (catalog no. A28526, Life Technologies) on the Applied Biosystems (AB) 7500 Fast Dx Real-Time PCR instrument. All rRT-PCR reactions performed had a total reaction volume of $25 \mu \mathrm{L}$ with primer and probe reaction concentrations at $0.8 \mu \mathrm{M}$ and $0.2 \mu \mathrm{M}$, respectively. Thermocycling rRT-PCR conditions were as follows: $50^{\circ} \mathrm{C}$ for $30 \mathrm{~min}, \mathrm{Taq}$ activation at $95^{\circ} \mathrm{C}$ for $2 \mathrm{~min}$ and 45 cycles of $95^{\circ} \mathrm{C}$ for $15 \mathrm{~S}$ and $55^{\circ} \mathrm{C}$ for $30 \mathrm{~s}$. All analytical performance data were collected on an $A B I 7500$ Fast Dx Real-time PCR instrument. Increases in fluorescent signal were registered during the annealing step of the reaction. All data were analysed with the sequence detector software (SDS) v1.4.1 (Life Technologies).

\section{Analytical sensitivity and specificity}

In order to demonstrate rRT-PCR performance of the VIC deletion assay, five B/VIC lineage viruses and two $B / Y A M$ lineage viruses from recent circulating genetic groups, including the WHO-recommended influenza $B$ vaccine viruses $B / C o l o r a d o / 6 / 2017$ (V1A-2DEL), B/ Brisbane/60/2008 (V1A) and B/Phuket/3073/2013 (B/Yam) were selected for assay evaluation (Figure).
Analytical sensitivity of the Vic 2_Del and Vic No_Del assays was determined using two V1A-2DEL and $V_{1} A$ viruses. The Vic 3_Del assay was evaluated by using the V1A-3DEL virus B/Hong Kong/269/2017 and B/ Washington/02/2019. The sensitivity of the three assays was evaluated with the commercially available Invitrogen SuperScript III Platinum One-Step qRT-PCR kit.

The analytical sensitivity and specificity of the Vic deletion assay were evaluated by comparison with the performance of the universal influenza B assay $(\operatorname{InfB})$ from the CDC Flu rRT-PCR Dx Panel. The InfB assay is designed for detection of the nonstructural (NS) gene in all influenza $B$ viruses by targeting highly conserved regions of the NS gene. A quantified synthetic RNA material (Armored RNA Quant CDC-9, AsuraGen, Inc., Austin, US) containing sequences from the NS gene region derived from B/Colorado/o6/2017 (EPI 969380) was tested in triplicate to determine the minimum RNA copy number detectable by the InfB assay.

We further assessed the analytical specificity using these five $B / V I C$ lineage deletion viruses and two $B /$ YAM lineage viruses, 24 seasonal influenza $A$ and two avian influenza $A$ viruses.

\section{Assay performance on clinical specimens}

To demonstrate the performance of the Vic deletion assay against clinical specimens, we tested 167 clinical specimens, including $67 \mathrm{~B} / \mathrm{VIC}$-positive and $30 \mathrm{~B} / \mathrm{YAM}$ positive to demonstrate the assay's clinical sensitivity, as well as 30 seasonal influenza A-positive samples and 30 influenza-negative samples as determined by the CDC rRT-PCR Flu Panel to demonstrate the assay's clinical specificity.

\section{Ethical statement}

The specimens evaluated were collected in the course of public health surveillance and human subjects' approval was not required for this project.

\section{Results}

\section{Antigenic analysis}

Influenza $B$ viruses from the $V_{1} A, V_{1} A-2 D E L, V_{1} A-3 D E L$ and Yam lineages were antigenically characterised in $\mathrm{HI}$ tests using post-infection ferret sera raised against representative human influenza $B$ viruses. The $\mathrm{HI}$ assays demonstrated that both the $V_{1} A-2 D E L$ and the $V_{1} A-3 D E L$ variant influenza $B$ viruses were antigenically distinct from each other and from the no-deletion $B /$ VIC virus $\left(V_{1} A\right)$, as the viruses from each of the genetic groups were not well inhibited by antiserum raised against the other genetic groups (reductions in $\mathrm{HI}$ titres of 4-32-fold compared with the homologous $\mathrm{HI}$ titres) (Table 2).

\section{Real-time RT-PCR assay establishment}

The Vic deletion assay was developed using the $A B I$ 7500 Fast Dx Real-time PCR system for the qualitative 
TABLE 6

Clinical performance of the influenza B/Victoria lineage deletion detection assay: detection of other subtype/ lineage influenza specimens compared with the CDC Human Influenza Virus Real-Time RT-PCR Diagnostic Panel (CDC Flu rRT-PCR Dx Panel)

\begin{tabular}{|l|c|c|}
\hline \multirow{2}{*}{ CDC Flu rRT-PCR Dx Panel result } & \multicolumn{2}{|c|}{ B/VIC deletion assay ${ }^{2}$} \\
\cline { 2 - 3 } & Positive & Negative \\
\hline Influenza A (H1N1)pdmo9 and $\left(\mathrm{H}_{3} \mathrm{~N}_{2}\right)$ & 0 & $30(15+15)$ \\
\hline Influenza A and B-negative & 0 & 30 \\
\hline Influenza B/YAM & 0 & 30 \\
\hline
\end{tabular}

CDC: United States Centers for Disease Control and Prevention; Vic: Victoria; YAM: Yamagata.

a Including Vic No_Del, Vic 2_Del and Vic 3_Del targets.

detection and characterisation of B/VIC virus RNA in respiratory specimens from patients presenting with influenza-like illness (ILI).

We designed three influenza B/VIC genetic group-specific probes to target the deletion region of aa position 162-164 (corresponding to nt position 529-537) of the $\mathrm{HA}$ gene in influenza $B$ viruses (Table 1). The sequence surrounding the deletion contains multiple adenines and repeating sequences, creating a challenge for designing probes for this region. We found that unmodified RT-PCR probes were unsuccessful in targeting the deletion region owing to challenges in the surrounding sequence patterns (data not shown). BHQplus probes contain stabilising chemistry that allows a probe oligonucleotide to be a shorter length which more easily targets regions with challenging sequence patterns [21]. We used BHQplus chemistry to successfully design probes to the $V_{1} A$ and $V_{1} A-2 D E L$ influenza $B$ viruses (Table 1). When designing a probe for detection of the V1A-3DEL viruses, we attempted both a BHQplus design and a minor groove binding (MGB) probe for increased sequence specificity, but neither of these probe modifications were successful (data not shown). The LNA have been shown to increase stability and sequence mismatch detection $[17,18]$, and we found that using a triplet of LNA centred on the mismatch allowed for successful detection of the $\mathrm{V}_{1} \mathrm{~A}-3 \mathrm{DEL}$ viruses.

\section{Analytical sensitivity}

Analytical performance studies, evaluated by testing 10 -fold serial dilutions of RNA extracted from recently circulating influenza $B$ viruses, demonstrated that the sensitivity of the VIC 2_Del, VIC 3_Del and VIC No_Del assays was comparable to the InfB assay of the CDC Flu rRT-PCR Dx Panel.

Analytical sensitivity of the Vic deletion assay is shown in Table 3 and Supplementary Table S2. Three quantified B/VIC viruses B/Maryland/15/2016 (V1A2DEL), B/Hong Kong/269/2017 (V1A-3DEL) and B/ Florida/103/2016 ( $\left.V_{1} A\right)$ were used to determine the limit of detection of the Vic 2_Del, Vic 3_Del and Vic No_Del assays, respectively. The results demonstrated that the sensitivity of Vic 2_Del and Vic No_Del assays were comparable to InfB assay, with $<3$ cycle thresholds ( $\mathrm{Ct}$ ) difference between the InfB assay and the positive target of the Vic deletion assay. The Vic 3_Del assay was slightly less sensitive than the InfB assay, as the Ct value of Vic 3_Del probe was $3 \mathrm{Ct}$ above the InfB target in two dilutions of B/Hong Kong/269/2017 (Table 3).

The limits of detection of Vic 2_Del, Vic 3_Del and Vic No_Del assays were $10^{1.5}, 10^{-0.2}$ and $10^{0.3} \quad \mathrm{ID}_{50} /$ $\mathrm{mL}$, respectively $\left(10^{-0.2 \sim 1.5} \mathrm{ID} \mathrm{D}_{50} / \mathrm{mL}\right)$. This correlates to $10^{-2.5 \sim-0.8} I D_{50}$ per reaction $(5.0 \mu \mathrm{L} /$ reaction). To understand how many copies of RNA per reaction the deletion assay will detect, we first used Armored RNA Quant CDC-9-containing sequences from the NS gene region derived from $B / C o l o r a d o / 06 / 2017$ to evaluate the InfB assay. We then matched rRT-PCR results of the serial dilution of virus titres to RNA copies based on comparable $\mathrm{Ct}$ values in the InfB assay. Given this comparison, we estimate that the Vic 2_Del and Vic No_Del probes will detect five RNA copies per reaction and the Vic 3_Del probe will detect ca 50 RNA copies per reaction. Therefore, the sensitivity of the Vic deletion assay was ca 5-50 RNA copies per reaction (Table 3). Two additional $\mathrm{V}_{1} \mathrm{~A}$ viruses, $\mathrm{B} /$ Colorado/06/2017 (V1A-2DEL) and $B /$ Brisbane/60/2008 ( $\left.V_{1} A\right)$, were used to evaluate the Vic deletion assay within the lower virus titres, and similar sensitivity was observed (Supplementary Table S2).

We also developed a triplex rRT-PCR assay with one pair of conserved primers with FAM-labelled Vic 3_Del probe, Hex-labelled Vic 2_Del probe and CAL Fluor Red 610-labelled Vic No_Del probe. This triplex rRT-PCR Vic deletion assay showed the same level of sensitivity and specificity as the singleplex assays in detecting and discriminating the $\mathrm{B} / \mathrm{VIC}$ deletion variant viruses (Supplementary Table S2).

\section{Analytical specificity (exclusivity)}

We evaluated analytical exclusivity with high-titre influenza B VIC and YAM lineage viruses (Table 4). We did not observe any cross-reactivity when $V_{1} A$ viruses were tested with the VIC 2_Del and VIC 3_Del assays. Specificity was also demonstrated in $\mathrm{V}_{1} \mathrm{~A}-3 \mathrm{DEL}$ viruses tested with the VIC 2_Del assay and vice versa. We did not observe cross-reactivity from the VIC No_Del assay when used to test $V_{1} A-2 D E L$ and $V_{1} A-3 D E L$ viruses. Likewise, the three assays did not react with the $B /$ YAM lineage viruses tested (Table 4).

In order to demonstrate the absence of cross-reactivity with influenza A viruses subtypes, we performed exclusivity testing by examining 12 isolates each of contemporary seasonal influenza $A\left(\mathrm{H}_{3} \mathrm{~N}_{2}\right)$ and $A\left(\mathrm{H}_{1} \mathrm{~N}_{1}\right)$ pdmog viruses as well as the benchmark highly pathogenic avian influenza (HPAl) $\mathrm{A}\left(\mathrm{H}_{5} \mathrm{~N}_{1}\right)$ strain, A/ Vietnam/1203/2004, and Asian lineage avian influenza $\mathrm{A}\left(\mathrm{H}_{7} \mathrm{~N} 9\right)$ virus, $\mathrm{A} / \mathrm{Anhui} / 01 / 2013$. All influenza $\mathrm{A}$ viruses 
were negative in all three assays (Supplementary Table S3).

\section{Clinical performance of influenza B/Victoria lineage deletion detection assay}

To evaluate the clinical performance of the Vic deletion assay, we tested the assay on clinical specimens received at the CDC during influenza surveillance. The Vic deletion assay was successful in classifying the 77 $B / V I C$ clinical specimens into their respective genetic groups (30 V1A-like, $27 \mathrm{~V}$ 1A-2DEL-like and $20 \mathrm{~V} 1 \mathrm{~A}$ 3 DEL-like) and, the rRT-PCR results were confirmed by sequencing (Table 5). All 30 influenza B/YAM, 15 each of influenza $A\left(\mathrm{H}_{1} \mathrm{~N}_{1}\right)$ pdmog and $A\left(\mathrm{H}_{3} \mathrm{~N}_{2}\right)$, as well as 30 influenza $A$ and $B$-negative samples tested negative for all B/VIC deletion assay targets (Table 6).

\section{Discussion}

The Vic deletion assay presented here is intended for the qualitative detection of the influenza $B / \mathrm{VIC}$ lineage HA gene deletion variant viruses using rRT-PCR technology. The analytical and clinical performance of the Vic deletion assay in either the singleplex or triplex configuration demonstrates that the assay is highly efficient, sensitive and comparable to the gold standard CDC rRTPCR Flu Panel InfB assay [20]. We have further shown that the primers and probes of the VIC deletion assay are specific to $V_{1} A, V_{1} A-2 D E L$ and $V_{1} A-3 D E L$ viruses and do not cross-react with $B / Y A M$ viruses, seasonal influenza $A$, or avian influenza $A$ viruses including HPAI $A\left(\mathrm{H}_{5} \mathrm{~N}_{1}\right)$ and $A$ sian lineage $A\left(\mathrm{H}_{7} \mathrm{~N} 9\right)$ influenza viruses.

The key genetic distinction between $V_{1} A, V_{1} A-2 D E L$, and $V_{1} A-3 D E L$ viruses is within the same nucleic acid region, presenting a challenge for $\mathrm{rRT}$-PCR probe design. We used two chemical modifications in probe design, LNA and BHQplus chemistry, which allowed for greater stability and specificity. Including LNA in real-time PCR probes improves detection of mismatches significantly $[16,17]$. The Vic 3_Del probe was designed and optimised using a triplet LNA approach, as probes labelled with a triplet of LNA residues centred on the mismatch provide greater discriminatory power than probes with a single LNA modification [17,18]. The probes for $V_{1} A$ and $\mathrm{V}_{1} \mathrm{~A}-2 \mathrm{DEL}$ were designed using BHQplus chemistry, allowing for stabilisation and enhanced mismatch detection with an overall shorter probe length [21]. We found that unmodified probes were not sufficient for this assay.

Sequence alignments using $\mathrm{HA}$ gene sequences from 2010 to 2019 demonstrated the conserved primers to be stable with no conserved genetic changes observed (data not shown). Although the areas chosen for the conserved amplification primers and the deletion typespecific probes are currently stable, genetic changes following rapid virus evolution and the variable nature of RNA viruses may require periodic updates of the Vic deletion assay primer and probe sequences.
The number and percentage of circulating $V_{1} A-2 D E L$ and $V_{1} A-3 D E L$ viruses has increased significantly since initial identification [22], making it critical to have a simplified and sensitive assay capable of detecting these genetic variants during influenza surveillance. The Vic deletion rRT-PCR assay is the most sensitive method currently available that can distinguish these influenza B genetic subgroups. One limitation of our assay is that it cannot distinguish between the two antigenically distinct groups within the $V_{1} A-3 D E L$ viruses (V1A.2 and $V_{1} A .3$ ), but it does detect $V_{1} A-3 D E L$ viruses, regardless of antigenicity. Recently, a pyrosequencing method was published that can also be used to distinguish these influenza $B$ genetic variants [11]. However, the pyrosequencing method requires costly instruments that are not common to diagnostic laboratories, and rRT-PCR is more sensitive than sequencing-based methods [23,24]. A conventional RT-PCR assay has also recently been developed to distinguish these influenza B genetic subgroups, but conventional RT-PCR is less sensitive and more time-consuming than an rRT-PCR assay [12].

\section{Conclusion}

Given its sensitivity and specificity, the rapidity of results, the fact that the Vic deletion assay can be run on equipment that is readily available in most diagnostic laboratories and the fact that the assay can be multiplexed for testing efficiency and cost-savings, this is a suitable assay for influenza surveillance. Combining the CDC Vic deletion assay with the CDC Flu rRT-PCR Dx Panel Influenza B Lineage Genotyping Kit will allow for rapid identification of the distinct influenza $B$ viral genetic groups $B / Y A M, B / V_{I C} V_{1} A, V_{1} A-2 D E L$ and $V_{1} A$ 3DEL [19]. This will further our understanding of the health impact of each of these antigenically and genetically distinct viruses in different global regions, which in turn will contribute to the most relevant vaccine virus recommendations moving forward into future influenza seasons.

\section{${ }^{\star}$ Erratum}

In the pdf version of this article, the ID50 values in Tables 3 and 4 were incorrectly printed without superscripts. This mistake has not affected the online html version of the article. The pdf was corrected on 22 October. We apologise for the error.

Acknowledgements

We thank the authors, originating and submitting laboratories of the sequences from GISAID's EpiFlu Database.

Funding: This work was supported by the Centers for Disease Control and Prevention.

Conflict of interest

None declared. 
Chickasaw Nation Industries, Inc. (CNI) is a federally chartered corporation wholly owned by the Chickasaw Nation. Headquartered in Norman, Oklahoma, CNI serves as a holding company with multiple subsidiaries that operate as Limited Liability Companies (LLCs) engaged in several lines of business including Civilian, Defense, Filtration, Network Services, and Research, Development, Test and Evaluation.

Battelle, headquartered in Columbus, $\mathrm{OH}$, conducts research and development, designs and manufactures products, and delivers services for the national security, health and life sciences, and energy and environmental industries.

The findings and conclusions in this report are those of the authors and do not necessarily represent the views of the Centers for Disease Control and Prevention or the Agency for Toxic Substances and Disease Registry.

\section{Authors' contributions}

BS designed the study, which was supervised by JRB and SL. BS and MKK wrote the manuscript and performed preliminary assay evaluation. MKK, WMD, JL and MMW performed the clinical tests. CW isolated and qualified reference viruses used for development of the diagnostic assay. WMS performed the haemagglutination inhibition tests. DEW and JRB edited the manuscript

\section{References}

1. Yamashita M, Krystal M, Fitch WM, Palese P. Influenza B virus evolution: co-circulating lineages and comparison of evolutionary pattern with those of influenza $A$ and $C$ viruses. Virology. 1988;163(1):112-22. https://doi.org/10.1016/00426822(88)90238-3 PMID: 3267218

2. McCullers JA, Saito T, Iverson AR. Multiple genotypes of influenza B virus circulated between 1979 and 2003. J Virol. 2004;78(23):12817-28. https://doi.org/10.1128/ JVI.78.23.12817-12828.2004 PMID: 15542634

3. Houser K, Subbarao K. Influenza vaccines: challenges and solutions. Cell Host Microbe. 2015;17(3):295-300. https://doi. org/10.1016/j.chom.2015.02.012 PMID: 25766291

4. Bennett J, Dolin R, Blaser MJ. Mandell, Douglas, and Bennett's principles and practices of infectious diseases. 8th edition. Philadelphia: Elsevier; 2016.

5. Rota PA, Wallis TR, Harmon MW, Rota JS, Kendal AP, Nerome K. Cocirculation of two distinct evolutionary lineages of influenza type B virus since 1983. Virology. 1990;175(1):59-68. https:// doi.org/10.1016/0042-6822(90)90186-U PMID: 2309452

6. Blanton L, Alabi N, Mustaquim D, Taylor C, Kniss K, Kramer N, et al. Update: influenza activity in the United States during the 2016-17 season and composition of the 2017-18 influenza vaccine. MMWR Morb Mortal Wkly Rep. 2017;66(25):668-76. https://doi.org/10.15585/mmwr.mm6625a3 PMID: 28662019

7. World Health Organization (WHO). Recommended composition of influenza virus vaccines for use in the 2018-2019 northern hemisphere influenza season. Geneva: WHO; 2018. Available from: http://www.who.int/influenza/vaccines/virus/ recommendations/201802_recommendation.pdf

8. World Health Organization (WHO). Recommended composition of influenza virus vaccines for use in the 2019-2020 northern hemisphere influenza season. Geneva: WHO; 2019. Available from: https://www.who.int/influenza/vaccines/virus/ recommendations/2019_20_north/en/

9. Shaw ML. PPO, In: Knipe DM H, PM, eds. Fields virology, 6th edition. Philadelphia: Lippincott Williams and Wilkins; 2013:1151-84.

10. Ambrose CS, Levin MJ. The rationale for quadrivalent influenza vaccines. Hum Vaccin Immunother. 2012;8(1):81-8. https://doi. org/10.4161/hv.8.1.17623 PMID: 22252006

11. Lau H, Deng YM, Xu X, Sessions W, Barr IG. Rapid detection

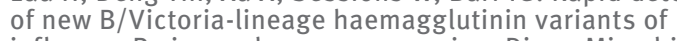
influenza B viruses by pyrosequencing. Diagn Microbiol Infect Dis. 2019;93(4):311-7. https://doi.org/10.1016/j. diagmicrobio.2018.11.003 PMID: 30528425
12. Chan WM, Wong LH, So CF, Chen LL, Wu WL, Ip JD, et al. Development and evaluation of a conventional RT-PCR for

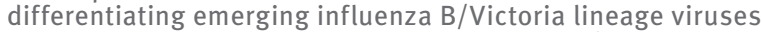
with hemagglutinin amino acid deletion from B/Yamagata lineage viruses. J Med Virol. 2020;92(3):382-5. https://doi. org/10.1002/jmv.25607 PMID: 31608480

13. Szretter KJ, Balish AL, Katz JM. Influenza: propagation, quantification, and storage. Curr Protoc Microbiol. 2006; Chapter 15:Unit 15G 1.

14. Reed LJ, Muench H. A simple method of estimating fifty percent endpoints. Am J Hyg. 1938;27:493-7.

15. Kendal AP, Cate TR. Increased sensitivity and reduced specificity of hemagglutination inhibition tests with ethertreated influenza B/Singapore/222/79. J Clin Microbiol. 1983;18(4):930-4. https://doi.org/10.1128/JCM.18.4.930934.1983 PMID: 6630472

16. Shu B, Wu KH, Emery S, Villanueva J, Johnson R, Guthrie $E$, et al. Design and performance of the CDC real-time reverse transcriptase PCR swine flu panel for detection of $2009 \mathrm{~A}\left(\mathrm{H}_{1} \mathrm{~N}_{1}\right)$ pandemic influenza virus. J Clin Microbiol. 2011;49(7):2614-9. https://doi.org/10.1128/JCM.02636-10 PMID: 21593260

17. Ballantyne KN, van Oorschot RA, Mitchell RJ. Locked nucleic acids in PCR primers increase sensitivity and performance. Genomics. 2008;91(3):301-5. https://doi.org/10.1016/j. ygeno.2007.10.016 PMID: 18164179

18. You Y, Moreira BG, Behlke MA, Owczarzy R. Design of LNA probes that improve mismatch discrimination. Nucleic Acids Res. 2006;34(8):e6o. https://doi.org/10.1093/nar/gkl175 PMID: 16670427

19. US Food and Drug Administration. CDC Human Influenza Virus Real-time RT-PCR Diagnostic Panel: Influenza A/B Typing Kit, Influenza A Subtyping Kit (VER 2), Influenza B Lineage Genotyping Kit (VER 1.1 and VER 2), Influenza A/H5 Subtyping Kit (VER 3). Atlanta: Centers for Disease Control and Prevention; 2019. Available from: https://www.accessdata.fda. gov/cdrh_docs/pdf19/K190302.pdf

20. Centers for Disease Control and Prevention (CDC). Centers for Disease Control and Prevention human influenza virus real-time RT-PCR detection and characterization pane. Atlanta: CDC; 2008. Available from: https://www.accessdata.fda.gov/ cdrh docs/pdf8/k080570.pdf

21. Sowers BA, Messenger VE, Cook RM. Genotyping with BHOplusTM fortified probes achieve powerful discrimination. Palo Alto, CA: qPCR Symposium. 2007. Available from: https:// biosearchassets.blob.core.windows.net/assets/bti_BHQplus_ Genotyping.pdf

22. Chow EJ, Davis CT, Abd Elal Al, Alabi N, Azziz-Baumgartner E, Barnes J, et al. Update: influenza activity - United States and worldwide, May 20-October 13, 2018. MMWR Morb Mortal Wkly Rep. 2018;67(42):1178-85. https://doi.org/10.15585/mmwr. mm6742a3 PMID: 30359347

23. Xiang Z, Wan R, Zou B, Qi X, Huang Q, Kumar S, et al. Highly sensitive and specific real-time PCR by employing serial invasive reaction as a sequence identifier for quantifying EGFR mutation abundance in cfDNA. Anal Bioanal Chem. 2018;410(26):6751-9. https://doi.org/10.1007/s00216-0181316-Z PMID: 30128808

24. Khot PD, Fredricks DN. PCR-based diagnosis of human fungal infections. Expert Rev Anti Infect Ther. 2009;7(10):1201-21. https://doi.org/10.1586/eri.09.104 PMID: 19968513

\section{License, supplementary material and copyright}

This is an open-access article distributed under the terms of the Creative Commons Attribution (CC BY 4.0) Licence. You may share and adapt the material, but must give appropriate credit to the source, provide a link to the licence and indicate if changes were made.

Any supplementary material referenced in the article can be found in the online version.

This article is copyright of the authors or their affiliated institutions, 2020. 